\title{
A Note on the Asymptotic Behavior of Parabolic Monge-Ampère Equations on Riemannian Manifolds
}

\author{
Qiang Ru \\ Center of Mathematical Sciences, Zhejiang University, Hangzhou 310027, China \\ Correspondence should be addressed to Qiang Ru; ruqiang666@yahoo.cn
}

Received 8 November 2012; Accepted 21 February 2013

Academic Editor: Junjie Wei

Copyright (C) 2013 Qiang Ru. This is an open access article distributed under the Creative Commons Attribution License, which permits unrestricted use, distribution, and reproduction in any medium, provided the original work is properly cited.

We study the asymptotic behavior of the parabolic Monge-Ampère equation $\partial \varphi(x, t) / \partial t=\log (\operatorname{det}(g(x)+\operatorname{Hess} \varphi(x, t)) / \operatorname{det} g(x))-$ $\lambda \varphi(x, t)$ in $\mathbb{M} \times(0, \infty), \varphi(x, 0)=\varphi_{0}(x)$ in $\mathbb{M}$, where $\mathbb{M}$ is a compact complete Riemannian manifold, $\lambda$ is a positive real parameter, and $\varphi_{0}(x): \mathbb{M} \rightarrow \mathbb{R}$ is a smooth function. We show a meaningful asymptotic result which is more general than those in Huisken, 1997.

\section{Introduction}

The main purpose of this paper is to study the asymptotic behavior of the parabolic Monge-Ampère equation:

$$
\begin{gathered}
\frac{\partial \varphi(x, t)}{\partial t}=\log \left\{\frac{\operatorname{det}(g(x)+\operatorname{Hess} \varphi(x, t))}{\operatorname{det} g(x)}\right\} \\
-\lambda \varphi(x, t) \quad \text { in } \mathbb{M} \times(0, \infty), \\
\varphi(x, 0)=\varphi_{0}(x), \quad \text { in } \mathbb{M},
\end{gathered}
$$

where $\mathbb{M}$ is a compact complete Riemannian manifold, $\lambda$ is a positive real parameter, and $\varphi_{0}(x): \mathbb{M} \rightarrow \mathbb{R}$ is a smooth function. We show a meaningful precisely asymptotic result which is more general than those in [1].

Monge-Ampère equations arise naturally from some problems in differential geometry. The existence and regularity of solutions to Monge-Ampère equations have been investigated by many mathematicians [1-8]. The long time existence and convergence of solution to (1) have been investigated in [1]. To some extent, we extend asymptotic result obtained in [1] in this paper. Hence, our main result is following analogue of Theorem 1.2 of [1].
Theorem 1. Let $\varphi$ be the solution of (1) with $\lambda>0$. For $p>1$, there exists $\delta>0$ and $f>0$ depending on $\varphi_{0}$ and $\left\|\nabla^{\beta} \varphi\right\|_{L^{\infty}}(\beta=0,1,2,3)$ such that

$$
\begin{aligned}
& \int_{\mathbb{M}}\left(\varphi^{p}-\overline{\varphi^{p}}\right)^{2} d \mu \\
& \quad \leq f \exp \left(-2\left[\frac{(2 p-1) \eta_{1}}{p}+p \lambda-\varepsilon(t)\right] t\right),
\end{aligned}
$$

where $\overline{\varphi^{p}}$ denotes the mean value of $\varphi^{p}, \eta_{1}$ is the first eigenvalue of the Laplacian, and $\varepsilon(t) \stackrel{\Delta}{=} \exp (-\delta t)$.

Remark 2. If $p=1$, Theorem 1 is in accordance with Theorem 1.2 of $[1]$.

Lemma 3 (see [1]). There exists positive constants $C_{0}\left(\varphi_{0}, \lambda\right)$ and $C_{1}$ depending on $(\mathbb{M}, g), \varphi_{0},\|\dot{\varphi}\|_{L^{\infty}},\left\|\nabla^{\beta-\gamma} \varphi\right\|_{L^{\infty}}(\beta=$ $0,1,2,3 ; \gamma=1,2,3 ; \gamma \leq \beta)$ such that

$$
\begin{aligned}
& |\dot{\varphi}| \leq C_{0} \exp (-\lambda t), \\
& \left|\nabla^{\beta} \varphi\right|^{2} \leq C_{1} \exp (-2 \lambda t) .
\end{aligned}
$$

Theorem 1 is proved in Section 2. 


\section{Asymptotic Behavior}

Proof of Theorem 1. In local coordinates, we have the following evolution equation:

$$
\begin{aligned}
\frac{\partial \varphi}{\partial t}= & \log \left\{\frac{\operatorname{det}(g+\operatorname{Hess} \varphi)}{\operatorname{det} g}\right\}-\lambda \varphi \\
= & \Delta \varphi+\log \operatorname{det}\left(g_{i j}+\nabla_{i j} \varphi\right) \\
& -\log \operatorname{det} g_{i j}-\Delta \varphi-\lambda \varphi \\
= & \Delta \varphi+\int_{0}^{1} \frac{d}{d s}\left\{\log \operatorname{det}\left(g_{i j}+s \nabla_{i j} \varphi\right)\right. \\
& -(s-1) \Delta \varphi\} d s-\lambda \varphi .
\end{aligned}
$$

Now, setting

$$
\begin{gathered}
\bar{g}_{s}^{i j} \stackrel{\Delta}{=}\left(g_{i j}+s \nabla_{i j}\right)^{-1}, \\
A \stackrel{\Delta}{=} \iint_{0}^{1} s\left|\nabla^{2} \varphi\right|_{\bar{g}_{\alpha s}}^{2} d \alpha d s .
\end{gathered}
$$

We rewrite (4) in more convenient notation as

$$
\begin{aligned}
\frac{\partial \varphi}{\partial t}= & \Delta \varphi+\int_{0}^{1}\left(\bar{g}_{s}^{i j}-g^{i j}\right) \nabla_{i j} \varphi d s-\lambda \varphi \\
= & \Delta \varphi+\iint_{0}^{1} \frac{d}{d \alpha}\left(g_{i j}+\alpha s \nabla_{i j} \varphi\right)^{-1} \nabla_{i j} \varphi d \alpha d s-\lambda \varphi \\
= & \Delta \varphi-\iint_{0}^{1}\left(g_{i k}+\alpha s \nabla_{i k} \varphi\right)^{-1}\left(g_{j l}+\alpha s \nabla_{j l} \varphi\right)^{-1} \\
& \times s \nabla_{k l} \varphi \nabla_{i j} \varphi d \alpha d s-\lambda \varphi \\
= & \Delta \varphi-\iint_{0}^{1} s\left|\nabla^{2} \varphi\right|_{\bar{g}_{\alpha s}}^{2} d \alpha d s-\lambda \varphi \\
= & \Delta \varphi-A-\lambda \varphi .
\end{aligned}
$$

We want to apply Gronwall inequality and hence consider the following equation:

$$
\begin{aligned}
\frac{\partial}{\partial t} \int_{\mathbb{M}} & \left(\varphi^{p}-\overline{\varphi^{p}}\right)^{2} d \mu \\
= & 2 p \int_{\mathbb{M}}\left(\varphi^{p}-\overline{\varphi^{p}}\right) \varphi^{p-1} \dot{\varphi} d \mu \\
= & 2 p \int_{\mathbb{M}}\left(\varphi^{p}-\overline{\varphi^{p}}\right) \varphi^{p^{-1}}(\Delta \varphi-A-\lambda \varphi) d \mu \\
= & 2 p \int_{\mathbb{M}}\left(\varphi^{p}-\overline{\varphi^{p}}\right) \varphi^{p-1} \Delta \varphi d \mu \\
& -2 p \int_{\mathbb{M}}\left(\varphi^{p}-\overline{\varphi^{p}}\right) \varphi^{p-1} A d \mu \\
& -2 p \lambda \int_{\mathbb{M}}\left(\varphi^{p}-\overline{\varphi^{p}}\right) \varphi^{p} d \mu .
\end{aligned}
$$

Notice that

$$
\int_{\mathbb{M}} \overline{\varphi^{p}}\left(\varphi^{p}-\overline{\varphi^{p}}\right) d \mu=0
$$

We obtain

$$
-2 p \lambda \int_{\mathbb{M}}\left(\varphi^{p}-\overline{\varphi^{p}}\right) \varphi^{p} d \mu=-2 p \lambda \int_{\mathbb{M}}\left(\varphi^{p}-\overline{\varphi^{p}}\right)^{2} d \mu .
$$

Furthermore we have

$$
\begin{aligned}
& 2 p \int_{\mathbb{M}}\left(\varphi^{p}-\overline{\varphi^{p}}\right) \varphi^{p-1} \Delta \varphi d \mu \\
& =-2 p \int_{\mathbb{M}} \nabla\left\{\left(\varphi^{p}-\overline{\varphi^{p}}\right) \varphi^{p-1}\right\} \nabla \varphi d \mu \\
& =-2 p \int_{\mathbb{M}}\left\{\nabla\left(\varphi^{p}-\overline{\varphi^{p}}\right) \varphi^{p-1}\right. \\
& \left.+(p-1) \varphi^{p-2} \nabla \varphi\left(\varphi^{p}-\overline{\varphi^{p}}\right)\right\} \nabla \varphi d \mu \\
& =-2 \int_{\mathbb{M}}\left|\nabla\left(\varphi^{p}-\overline{\varphi^{p}}\right)\right|^{2} d \mu-\frac{2(p-1)}{p} \\
& \times \int_{\mathbb{M}}\left|\nabla\left(\varphi^{p}-\overline{\varphi^{p}}\right)\right|^{2} d \mu \\
& +2 p(p-1) \overline{\varphi^{p}} \int_{\mathbb{M}} \varphi^{p-2}|\nabla \varphi|^{2} d \mu \\
& =-\frac{2(2 p-1)}{p} \int_{\mathbb{M}}\left|\nabla\left(\varphi^{p}-\overline{\varphi^{p}}\right)\right|^{2} d \mu \\
& +2 p(p-1) \overline{\varphi^{p}} \int_{\mathbb{M}} \varphi^{p-2}|\nabla \varphi|^{2} d \mu .
\end{aligned}
$$

We use the Poincare inequality

$$
\left\|\nabla\left(\varphi^{p}-\overline{\varphi^{p}}\right)\right\|_{L^{2}} \geq \eta_{1}\left\|\left(\varphi^{p}-\overline{\varphi^{p}}\right)\right\|_{L^{2}} .
$$

It follows that

$$
\begin{aligned}
2 p \int_{\mathbb{M}}\left(\varphi^{p}-\overline{\varphi^{p}}\right) \varphi^{p-1} \Delta \varphi d \mu \\
\leq-\frac{2(2 p-1) \eta_{1}}{p} \int_{\mathbb{M}}\left(\varphi^{p}-\overline{\varphi^{p}}\right)^{2} d \mu \\
\quad+2 p(p-1) \overline{\varphi^{p}} \int_{\mathbb{M}} \varphi^{p-2}|\nabla \varphi|^{2} d \mu .
\end{aligned}
$$

Moreover, we have that

$$
\begin{aligned}
& -2 p \int_{\mathbb{M}}\left(\varphi^{p}-\overline{\varphi^{p}}\right) \varphi^{p-1} A d \mu \\
& \quad=2 p \int_{0}^{1} \int_{\mathbb{M}}\left(\bar{g}_{s}^{i j}-g^{i j}\right) \nabla_{i j} \varphi\left(\varphi^{p}-\overline{\varphi^{p}}\right) \varphi^{p-1} d \mu
\end{aligned}
$$




$$
\begin{aligned}
& =-2 p \int_{0}^{1} \int_{\mathbb{M}} \nabla_{i}\left(\bar{g}_{s}^{i j}-g^{i j}\right) \nabla_{j} \varphi\left(\varphi^{p}-\overline{\varphi^{p}}\right) \varphi^{p-1} d \mu \\
& -2 p \int_{0}^{1} \int_{\mathbb{M}}\left(\bar{g}_{s}^{i j}-g^{i j}\right) \nabla_{j} \varphi \nabla_{i}\left(\varphi^{p}-\overline{\varphi^{p}}\right) \varphi^{p-1} d \mu \\
& -2 p \int_{0}^{1} \int_{\mathbb{M}}\left(\bar{g}_{s}^{i j}-g^{i j}\right) \nabla_{j} \varphi\left(\varphi^{p}-\overline{\varphi^{p}}\right) \nabla_{i}\left(\varphi^{p-1}\right) d \mu \\
& =-2 \int_{0}^{1} \int_{\mathbb{M}} \nabla_{i}\left(\bar{g}_{s}^{i j}-g^{i j}\right) \nabla_{j}\left(\varphi^{p}-\overline{\varphi^{p}}\right)\left(\varphi^{p}-\overline{\varphi^{p}}\right) d \mu \\
& -2 \int_{0}^{1} \int_{\mathbb{M}}\left(\bar{g}_{s}^{i j}-g^{i j}\right) \nabla_{j}\left(\varphi^{p}-\overline{\varphi^{p}}\right) \nabla_{i}\left(\varphi^{p}-\overline{\varphi^{p}}\right) d \mu \\
& -2 p(p-1) \int_{0}^{1} \int_{\mathbb{M}}\left(\bar{g}_{s}^{i j}-g^{i j}\right) \varphi^{2(p-1)} \nabla_{i} \varphi \nabla_{j} \varphi d \mu \\
& +2 p(p-1) \int_{0}^{1} \int_{\mathbb{M}}\left(\bar{g}_{s}^{i j}-g^{i j}\right) \varphi^{p-2} \nabla_{i} \varphi \nabla_{j} \varphi \overline{\varphi^{p}} d \mu \\
& =-2 \int_{0}^{1} \int_{\mathbb{M}} \nabla_{i}\left(\bar{g}_{s}^{i j}-g^{i j}\right) \nabla_{j}\left(\varphi^{p}-\overline{\varphi^{p}}\right)\left(\varphi^{p}-\overline{\varphi^{p}}\right) d \mu \\
& -\frac{2(2 p-1)}{p} \int_{0}^{1} \int_{\mathbb{M}}\left(\bar{g}_{s}^{i j}-g^{i j}\right) \nabla_{j}\left(\varphi^{p}-\overline{\varphi^{p}}\right) \nabla_{i} \\
& \times\left(\varphi^{p}-\overline{\varphi^{p}}\right) d \mu \\
& +2 p(p-1) \overline{\varphi^{p}} \int_{0}^{1} \int_{\mathbb{M}}\left(\bar{g}_{s}^{i j}-g^{i j}\right) \varphi^{p-2} \nabla_{i} \varphi \nabla_{j} \varphi d \mu \\
& \leq 2 \int_{0}^{1} \int_{\mathbb{M}}\left|\nabla\left(\bar{g}_{s}^{i j}-g^{i j}\right)\right| \cdot\left|\nabla\left(\varphi^{p}-\overline{\varphi^{p}}\right)\right| \cdot\left|\varphi^{p}-\overline{\varphi^{p}}\right| d \mu \\
& +\frac{2(2 p-1)}{p} \int_{0}^{1} \int_{\mathbb{M}}\left|\bar{g}_{s}^{i j}-g^{i j}\right| \cdot\left|\nabla\left(\varphi^{p}-\overline{\varphi^{p}}\right)\right|^{2} d \mu \\
& +2 p(p-1)\left|\overline{\varphi^{p}}\right| \int_{0}^{1} \int_{\mathbb{M}}\left|\left(\bar{g}_{s}^{i j}-g^{i j}\right)\right| \cdot\left|\varphi^{p-2}\right| \cdot|\nabla \varphi|^{2} d \mu \\
& \leq 2 C \sup \left|\nabla^{3} \varphi\right| \\
& \times\left(\int_{\mathbb{M}}\left|\nabla\left(\varphi^{p}-\overline{\varphi^{p}}\right)\right|^{2} d \mu+\int_{\mathbb{M}}\left(\varphi^{p}-\overline{\varphi^{p}}\right)^{2} d \mu\right) \\
& +\frac{2 C(2 p-1)}{p} \sup \left|\nabla^{2} \varphi\right| \int_{\mathbb{M}}\left|\nabla\left(\varphi^{p}-\overline{\varphi^{p}}\right)\right|^{2} d \mu \\
& +2 p(p-1) C\left|\overline{\varphi^{p}}\right| \sup \left|\nabla^{2} \varphi\right| \int_{\mathbb{M}}\left|\varphi^{p-2}\right| \cdot|\nabla \varphi|^{2} d \mu \text {. }
\end{aligned}
$$

where $C$ is always a constant that may change from line to line.

Substituting (9), (12), and (13) in the right-hand side of

$$
\begin{aligned}
\frac{\partial}{\partial t} \int_{\mathbb{M}} & \left(\varphi^{p}-\overline{\varphi^{p}}\right)^{2} d \mu \\
\leq & -2\left[\frac{(2 p-1) \eta_{1}}{p}+p \lambda\right] \int_{\mathbb{M}}\left(\varphi^{p}-\overline{\varphi^{p}}\right)^{2} d \mu \\
& +2 p(p-1) \overline{\varphi^{p}} \int_{\mathbb{M}} \varphi^{p-2}|\nabla \varphi|^{2} d \mu
\end{aligned}
$$

$$
\begin{aligned}
& +2 C \sup \left|\nabla^{3} \varphi\right|\left(\int_{\mathbb{M}}\left|\nabla\left(\varphi^{p}-\overline{\varphi^{p}}\right)\right|^{2} d \mu\right. \\
& \left.+\int_{\mathbb{M}}\left(\varphi^{p}-\overline{\varphi^{p}}\right)^{2} d \mu\right) \\
& +\frac{2 C(2 p-1)}{p} \sup \left|\nabla^{2} \varphi\right| \int_{\mathbb{M}}\left|\nabla\left(\varphi^{p}-\overline{\varphi^{p}}\right)\right|^{2} d \mu \\
& +2 p(p-1) C\left|\overline{\varphi^{p}}\right| \sup \left|\nabla^{2} \varphi\right| \int_{\mathbb{M}}\left|\varphi^{p-2}\right| \cdot|\nabla \varphi|^{2} d \mu .
\end{aligned}
$$

By Lemma 3, that is, the exponential decay of $\left|\nabla^{\beta} \varphi\right|_{L^{\infty}}(\beta=$ $0,1,2,3)$, it is easy to obtain the following.

For any $\varepsilon>0$, there exists a $T(\varepsilon)$ such that

$$
\begin{aligned}
\frac{\partial}{\partial t} \int_{\mathbb{M}}\left(\varphi^{p}-\overline{\varphi^{p}}\right)^{2} d \mu \\
\leq-2\left[\frac{(2 p-1) \eta_{1}}{p}+p \lambda-\varepsilon(t)\right] \\
\quad \times \int_{\mathbb{M}}\left(\varphi^{p}-\overline{\varphi^{p}}\right)^{2} d \mu \\
\quad+2 \varepsilon\left(\int_{\mathbb{M}}\left|\nabla\left(\varphi^{p}-\overline{\varphi^{p}}\right)\right|^{2} d \mu\right. \\
\left.\quad+\left|\overline{\varphi^{p}}\right| \int_{\mathbb{M}}\left|\varphi^{p-2}\right| \cdot|\nabla \varphi|^{2} d \mu\right) .
\end{aligned}
$$

The Gronwall inequality yields

$$
\begin{aligned}
& \int_{\mathbb{M}}\left(\varphi^{p}-\overline{\varphi^{p}}\right)^{2} d \mu \\
& \quad \leq f \exp \left(-2\left[\frac{(2 p-1) \eta_{1}}{p}+p \lambda-\varepsilon(t)\right] t\right),
\end{aligned}
$$

where the constant $f>0$ depending on $\varphi_{0}$ and $\left\|\nabla^{\beta} \varphi\right\|_{L^{\infty}}(\beta=$ $0,1,2,3)$.

Thus, the proof of Theorem 1 is completed.

\section{References}

[1] B. Huisken, "Parabolic Monge-Ampère equations on Riemannian manifolds," Journal of Functional Analysis, vol. 147, no. 1, pp. 140-163, 1997.

[2] T. Aubin, Nonlinear Analysis on Manifolds, Monge-Ampère Equations, vol. 252 of Fundamental Principles of Mathematical Sciences, Springerg, New York, NY, USA, 1982.

[3] P. Delanoë, "Équations du type Monge-Ampère sur les variétés riemanniennes compactes I," Journal of Functional Analysis, vol. 40, no. 3, pp. 358-386, 1981.

[4] P. Delanoë, "Équations du type de Monge-Ampère sur les variétés riemanniennes compactes II," Journal of Functional Analysis, vol. 41, no. 3, pp. 341-353, 1981.

[5] P. Delanoë, "Équations du type de Monge-Ampère sur les variétés riemanniennes compactes III," Journal of Functional Analysis, vol. 45, no. 3, pp. 403-430, 1982.

[6] L. Songzhe, "Existence of solutions to initial value problem for a parabolic Monge-Ampère equation and application," Nonlinear 
Analysis: Theory, Methods \& Applications, vol. 65, no. 1, pp. 5978, 2006.

[7] Z. T. Zhang and K. L. Wang, "Existence and non-existence of solutions for a class of Monge-Ampère equations," Journal of Differential Equations, vol. 246, no. 7, pp. 2849-2875, 2009.

[8] J. N. Huang and Z. W. Duan, "Existence of the global solution for the parabolic Monge-Ampère equations on compact Riemannian manifolds," Journal of Mathematical Analysis and Applications, vol. 389, no. 1, pp. 597-607, 2012. 


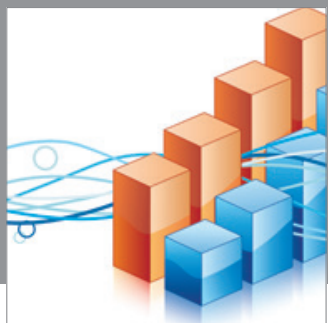

Advances in

Operations Research

mansans

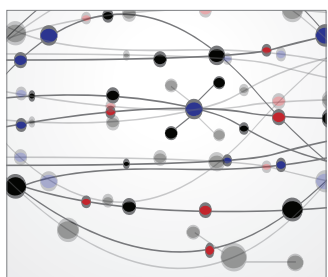

The Scientific World Journal
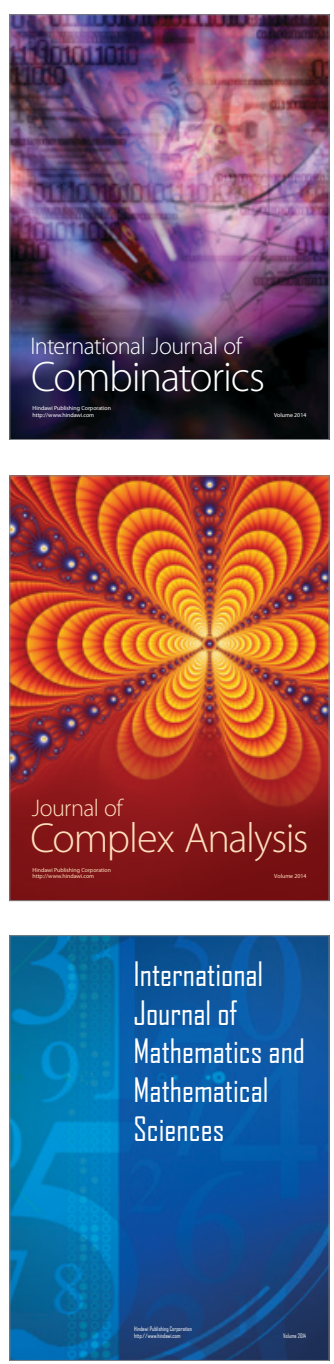
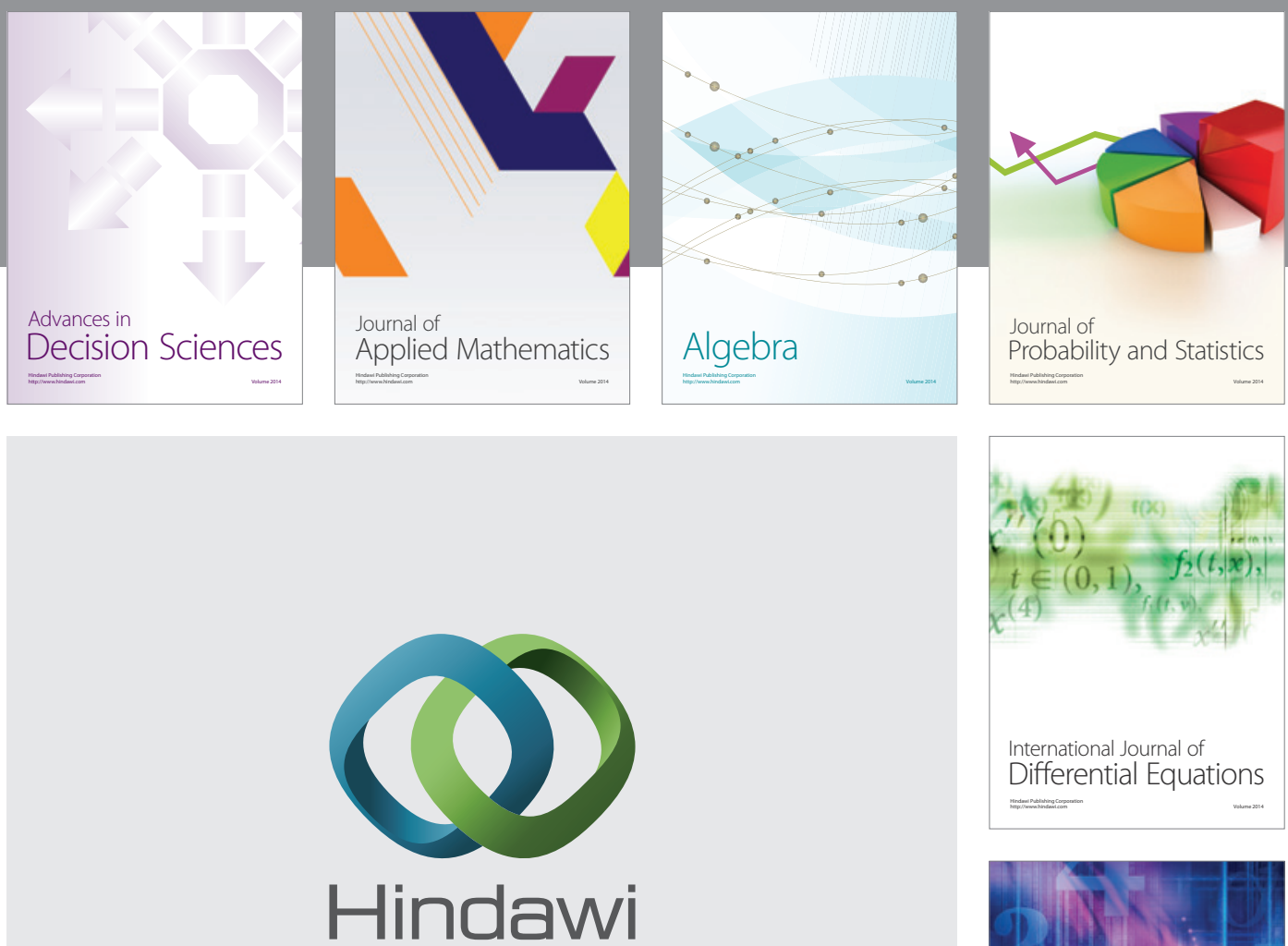

Submit your manuscripts at http://www.hindawi.com
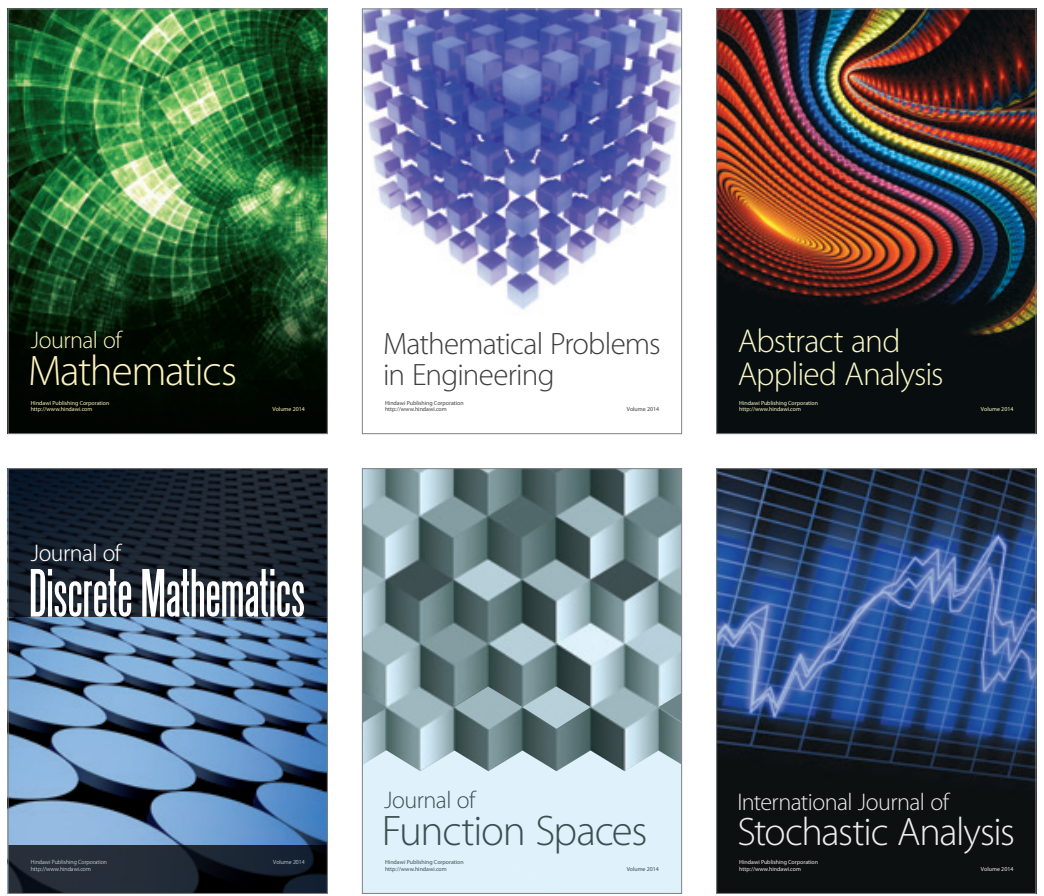

Journal of

Function Spaces

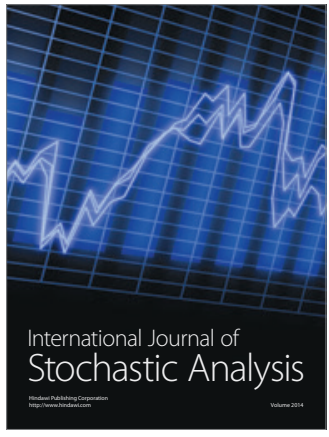

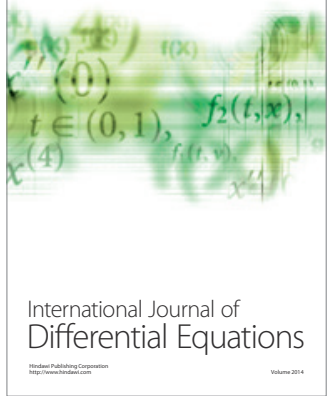
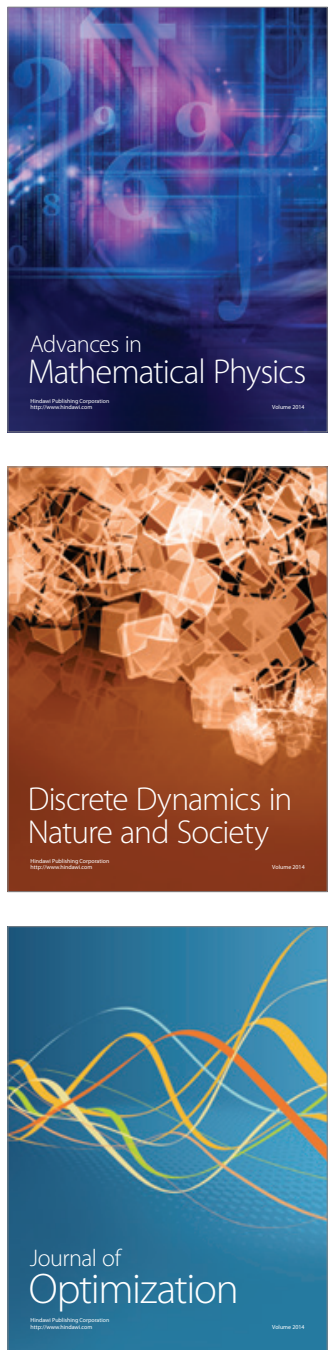